\title{
Validation of an ECG-Derived Respiration Monitoring Method
}

\author{
B Mazzanti ${ }^{1}$, C Lamberti ${ }^{1}, \mathrm{~J}$ de Bie $^{2}$ \\ ${ }^{1}$ DEIS University of Bologna Italy; ${ }^{2}$ Mortara Rangoni Europe, S.Giorgio di Piano, Bologna Italy
}

\begin{abstract}
The method proposed here includes automatic optimal lead(s) selection from multiple lead systems, calculates the EDR-values from QRS-area variations and obtains a respiratory waveform through interpolation. The respiratory frequency and the presence of apnea were calculated in the time-domain, identifying the respiratory cycles on the waveform through a min-max detection method.

Validation of the method has been performed using eleven patients affected mostly by obstructive respiration disorder in a sleep laboratory; gold standard respiration data was taken from oral/nasal flow, EDR was calculated using 12-lead ECG synchronized with the sleep data.

Summarized results for the automatic process are: Respiration cycle detection sensitivity/specificity was $98 \%$ / $90 \%$ respectively. Apnea detection sensitivity / specificity was $87 \% / 85 \%$ respectively.
\end{abstract}

\section{Introduction}

The measurement and monitoring of respiration is important in many circumstances. Respiration is the most important modulator of heart rate, and the source of the short term heart rate variability. Analysis of heart rate variability can give important insight in the autonomic regulation of heart rate, and the correlation with the input signal simplifies this analysis.

Respiration is also an important parameter in intensive care monitoring, and gradual increase of respiration rate is often reason for treatment changes.

Respiration disorder during sleep (Obstructive Sleep Apnea Syndrome) gives rise to significant problems in daily functioning, as well as being a risk factor for various cardiac diseases and associated with hypertension, right ventricular hypertrophy, heart failure and cardiovascular disease [7].

Several methods exist to measure respiration indirectly through thorax movements or electrical impedance variances. [6] The gold standard, however, is the direct measurement of air flow in the mouth and nose, usually through temperature measurement of in- and out flowing air.

The objective of the implementation of the ECG
Derived Respiration measurement method is to monitor respiration from an available ECG source, in the absence of any other separate signal. The method can be useful for the analysis of 24-hour ambulatory ECG recordings, in monitoring through telemetry, or in various other situations where other signals are not available.

The EDR method is based on small ECG morphology changes during the respiratory cycle caused by movement of the heart position relative to the electrodes and the change of lung volume (and therefore dielectric changes). The current method is mainly different from previous studies ([1-4]), others: see challenge. because 12-lead (8channel) ECG's are used, which has the advantage that it is possible to select the lead(s) that are most influenced by respiration. These are not always the classical leads that are used in 2 or 3 lead monitoring.

\section{Method}

\subsection{EDR}

The method of measuring the EDR-signal has been described more in details by Travaglini, $\mathrm{C}$ et al. ([5]). In short, the QRS area is calculated for every beat and all 8 independent leads. Area proved to be more stable and less prone to noise than amplitude measurements. Important is the subtraction of the ECG baseline value, taken immediately before the QRS complex. An ECG sample rate of 500 samples/s proved to be necessary for sufficient precision.

The first phase after starting monitoring is the learning phase. In this period, 60 subsequent cardiac cycles are taken (containing one or more full respiratory cycles), and the leads that contributed most to the presumed respiratory variations are estimated using a principal component decomposition. Stability of this choice was checked otherwise the procedure was repeated. In this way, either one or two adjacent leads were chosen for subsequent analysis. This method proved more stable and noise resistant then taking the linear combination of all 8 channels resulting from the principal component analysis.

After the learning period, the EDR value is calculated for every beat considered to be of "normal shape", and 
the resulting samples interpolated using a cubic spline for display purposes.

\subsection{Calculation of respiratory frequency and recognition of apnea}

Respiration frequency monitoring and apnea detecion are achieved by a time domain analysis of individual respiratory cycles of the EDR curve.

Individual peaks and valleys are detected first. Subsequently, peaks and valleys are discarded on the basis of duration and amplitude criteria:

- duration: a physiological minimum of $500 \mathrm{~ms}$ for a peak-to-valley distance was established in order to accepted as a respiratory waveform.

amplitude: in order to be acceptable, the amplitude difference between peak and valley had to be above a certain percentage of the average amplitude of the previous 10 respiratory cycles. This inter- and intraindividual adaptation proved to be necessary for a reliable performance.

These thresholds were set to values that gave good performance for respiration rate monitoring without missing too many apnea's.

Respiration rate was calculating in a moving 1 minute averaging.

Apnea causes a reduction in amplitude of the EDR waveform. However, thorax movements in the presence of obstructive apnea will inevitable cause some ECG morphology variations. The decision whether an episode is called apnea is based on:

- the variance of the episode with respect to the previous minute must be significantly lower

- the number of waves that are accepted as respiration waves (see previous paragraph) must be below a certain percentage of the total number of oscillations detected.

For the purpose of this study, the apnea duration threshold was set to $20 \mathrm{~s}$.

In certain circumstances, the calculation of the respiratory frequency and apnea was suspended (downtime), for the following reasons:

1. The presence of a "spike", erratic measurements of the QRS area, caused by noise on the ECG or sudden morphology changes.

2. Removal of an electrode or lead during the registration of the ECG

3. Under sampling of the EDR curve. Intrinsic of the method is the availability of a single respiration sample per valid QRS complex. If the heart rate is too low or too many beats are discarded, the respiration curve will be under sampled. We have used a threshold of 3 samples per respiratory cycle.

\subsection{Validation method}

Parallel data collection has been performed on patients during multi channel somnography. 12 lead high resolution (500 samples/s, $2.5 \mu \mathrm{V}$ LSB) ECG was transmitted to a computer with telemetry (Mortara Instrument X-12 transmitter). The somnography data of interest were exported to the same computer through the standard export tools of the equipment (Medcare/ FlagaMedical Embla system). These signals were: oralnasal air flow, thorax movements, abdominal movements and a single channel ECG signal. The data from the two sources was subsequently synchronized using a simultaneous trigger signal recorded on both ECG traces, and the signals from the somnograph were re-sampled at 500 samples/s for ease of processing and display.

Registrations were performed on 11 patients during routine sleep laboratory testing, mostly male, aged over 50 years, and all affected with the obstructed sleep apnea syndrome. Some were overweight as is typical of this type of population.

The duration of the recording varied, some were taken during the night and lasted over 6 hours, other were shorter afternoon sessions. For the purpose of this validation, one hour per patient was analysed, all in the beginning of the recording sessions.

The flow signal was used as the "truth", and the individual respiration waves and apnea's were annotated manually by the authors. These signals were clear-cut, without any doubt on the truth. Respiration rate was calculated every minute.

The high resolution ECG signal was processed by the algorithm described above, and the single respiration waveforms were compared with the truth, as well as the respiration rate and presence of apnea. Apnea threshold was defined as $20 \mathrm{~s}$.

The following parameters were calculated:

- Maximum per patient minute respiration rate difference

- Average per patient minute respiration rate difference

- Standard deviation of per patient minute respiration rate difference

- The number of true, detected and falsely detected apnea's per patient

- The algorithm down time per patient (excluding the initial learning period)

- Total population mean respiration error and maximum inter patient mean respiration rate error.

- Total respiration cycle detection specificity and sensitivity

- Total apnea detection sensitivity and specificity

- Total algorithm down time. 


\section{Results}

In all patients it was possible to calculate a consistent EDR signal and a reliable respiration frequency. All patients were in sinus rhythm and had normal width QRS complexes. There were some ventricular premature beats, but no runs or other arrhythmia's. The learning mechanism stabilized quickly in all recordings. Leads chosen by the algorithm were mostly anterior-lateral:

$\begin{array}{ll}\text { Leads } & \text { Cases } \\ \text { V2, V3 } & 1 \\ \text { V3, V4 } & 3 \\ \text { V4 alone } & 1 \\ \text { V4, V5 } & 3 \\ \text { V5, V6 } & 3\end{array}$

The figures demonstrate some of the resulting traces as a brief illustration. Scales are arbitrary.

Figure 1 shows an episode of a nocturnal registration, average heart rate $73 \mathrm{bpm}$ and a regular respiration. The EDR curve follows the true flow trace closely, apart from some amplitude and baseline fluctuations.

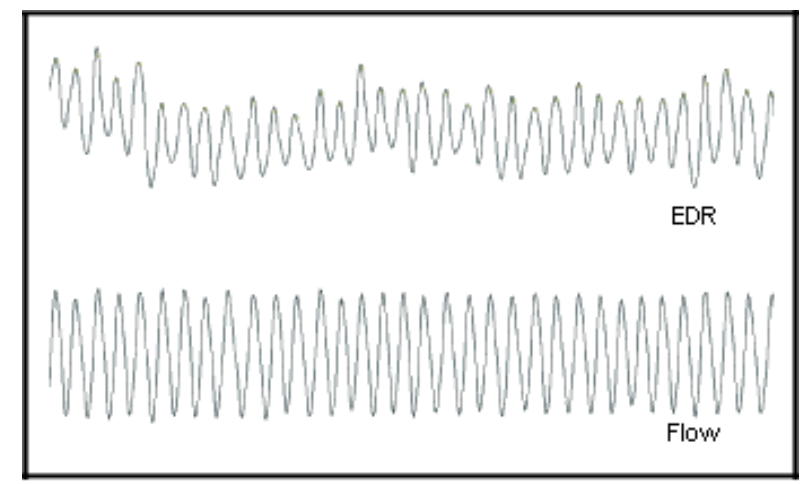

Figure 1. Example of EDR curve with normal regular respiration. Horizontal full scale 97 s, vertical scales arbitrary.

The second example is from a male 57 year old patient, height $160 \mathrm{~cm}$, weight $73 \mathrm{~kg}$, diagnosed with OSAS. He showed also frequent apnea, some with much reduced thorax and abdominal movements. Figure 2 shows an episode with various apnea's of short duration and the typical resulting EDR curve. The recognised apnea episodes are indicated on the EDR trace.

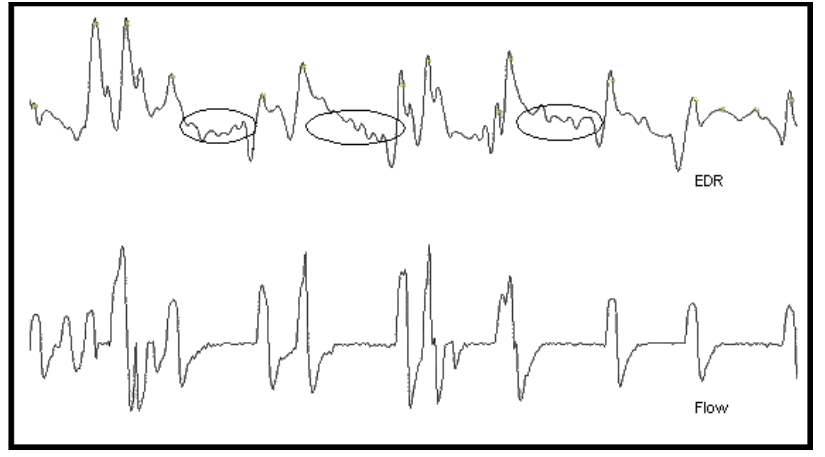

Figure 2. Example of registration of flow and EDR in the presence of irregular breathing and frequent apnea. Horizontal full scale $97 \mathrm{~s}$, vertical scales arbitrary. Identified apnea's are indicated.

Table 1 shows the per patient results. As can be seen, there is a slight under estimation of respiration frequency as a result of the compromise between adequate apnea detection and optimal respiration frequency calculation. The total population error EDR - Flow difference was $-0.3 \mathrm{rpm}$, with a maximum inter patient error of $3.5 \mathrm{rpm}$

Although standard deviation of the EDR-Flow difference is always below $2.5 \mathrm{rpm}$ (total population average $1.2 \mathrm{rpm}$ ), some single differences are above 5 rpm patient 4), which can give rise to false respiration rate alarms in real time monitoring.

The total respiration cycle recognition performance for the EDR method was measured in terms of $98 \%$ sensitivity and $90 \%$ specificity.

Worst performance was for patient 4 , who expressed a large number of apnea's.

Only three patients showed apnea, mostly of the obstructive type, maintaining chest and/or abdominal movements. Notwithstanding this, the EDR method was able to detect more than $85 \%$ of the events correctly without a too high false apnea rate. Apnea detection sensitivity was in fact $87 \%$ while specificity was $85 \%$.

The average down time was $4.5 \%$, with a maximum of $17 \%$ in one patient. Most of the down-time was casued by 3 patients. Main causes for the down-time were an episode of a radio transmission failure of the ECG (patient 5) and excessive noise on the ECG (patients 2 and 7); without these three patients, down time was only $1 \%$. 
Table 1. Summarized per patient results. All rates in rpm, apnea's in number of events, down time in percentage of total monitored time (excluding learning period).

\begin{tabular}{|c|c|c|c|c|c|c|c|c|c|c|c|}
\hline Patient & 1 & 2 & 3 & 4 & 5 & 6 & 7 & 8 & 9 & 10 & 11 \\
\hline $\begin{array}{l}\text { Maximum } \\
\text { EDR- Flow }\end{array}$ & -3.7 & -4.3 & -3.7 & 8.6 & -1.9 & 3.7 & -4.9 & 4.9 & 3.7 & -5.6 & -3.1 \\
\hline $\begin{array}{c}\text { Mean } \\
\text { EDR- Flow }\end{array}$ & -0.5 & -1.6 & -1.2 & 3.5 & -0.1 & 0.5 & -1.9 & -0.3 & 0.2 & -0.7 & -0.4 \\
\hline $\begin{array}{c}\text { ST. Dev. } \\
\text { EDR- Flow }\end{array}$ & 1.0 & 1.9 & 1.2 & 2.5 & 0.6 & 1.5 & 1.5 & 1.7 & 1.3 & 2.0 & 0.8 \\
\hline True Apnea's & - & - & - & 69 & - & 36 & 16 & - & 3 & - & - \\
\hline Detected Apnea's & - & - & - & 57 & - & 33 & 15 & - & 1 & - & - \\
\hline False Apnea's & - & 3 & - & 7 & - & 2 & 10 & - & - & - & - \\
\hline Down time (\%) & 2.6 & 13.1 & 3.3 & 0.0 & 16.8 & 0.2 & 11.6 & 0.4 & 0.3 & 0.4 & 1.0 \\
\hline
\end{tabular}

\section{Conclusions}

The intrinsic limits of the EDR method of measuring the respiration rate proved not to prevent it from being effective and did not discourage its use in this patient population. In addition it is an easy to implement method, also for Holter or telemetry data. As with methods using impedance or strain-gauges, the method by itself is not sufficient for OSAS recognition and reliable apnea monitoring. Non-obstructive apnea can probably be detected reliably, but not enough data was present in this set to confirm this.

Further validation on a larger patient group in coronary care intensive therapy is planned in the near future.

\section{Acknowledgements}

The authors wish to express their thanks to the sleep laboratories of the hospitals of the Maugeri foundation at Montescano (PV) and "Santa Maria della Misericordia" at Udine.

\section{References}

[1] Pinciroli F, Rossi R, Vergani L. Detection of electrical axis variation for the extraction of respiratory information. IEEE Proceedings of Computers in Cardiology 12:499502, 1985.

[2] Moody G, Mark R, Zoccola A, Mantero S, Derivation of respiratory signals from multi-lead ecg. IEEE Proceedings of Computers in Cardiology 12:113-116, 1985.

[3] Moody G, Mark R, Bump M, Weinstein J, Berman A, Mietus J, Goldberger A. Clinical validation of ecg derived respiration (edr) technique. IEEE Proceedings of Computers in Cardiology 13:507-510, 1986.

[4] Zhao L Reisman SFT. Derivation of respiration from electrocardiogram during heart rate variability studies. IEEE Proceedings of Computers in Cardiology 21:53-56, 1994.

[5] Travaglini A, Lamberti C ,De Bie J. Respiratory of signal derived from eight-lead ecg. IEEE Proceedings of Computers in Cardiology 25:2:65-68, 1998.

[6] Association for the Advancement of Medical Instrumentation. Apnea monitoring by means of thoracic impedance pneumography, 1989. Tecnical Iinformational Report 4:1989.

[7] Associazione Italiana di Medicina del Sonno. Aggiornamento monografico: Russamento ed apnea ostruttiva da sonno. January 2002

Address for correspondence.

Johan de Bie

Mortara Rangoni Europe srl

Via Oradour, 7

40017 San Giorgio di Piano (BO)

ITALY

E-mail: $\underline{\text { debie@mortara.com }}$ 